\title{
Intraoral and transcutaneous cervical ultrasound in the differential diagnosis of peritonsillar cellulitis and abscesses
}

Bernardo Cunba Araujo Filbo', Flavio A. Sakae ${ }^{2}$, Luiz Ubirajara Sennes ${ }^{3}$, Rui Imamura ${ }^{4}$, Marcus $R$. de Menezes ${ }^{5}$
Keywords: peritonsillar abscess, peritonsillar cellulitis, ultrasound.

\section{Summary}

\begin{abstract}
A
ims: The objective of the present study was to determine the specificity, sensitivity and accuracy of intraoral and transcutaneous ultrasound (US) in the diagnosis of peritonsillar cellulitis and abscess. Study Design: Clinical-Prospective. Materials and Metods: Thirty nine patients were seen at the otorhinolaryngology emergency department of the University Hospital, of the School of Medicine, University of São Paulo, with a clinical diagnosis of peritonsillar cellulitis or abscess. After initial evaluation, all patients were submitted to intraoral and transcutaneous US. Results: Intraoral US was performed on 35 cases and its sensitivity was of $95.2 \%$, the specificity was of $78.5 \%$ and the accuracy was of $86.9 \%$. Transcutaneous US was feasible in all 39 patients and diagnosed peritonsillar abscess in $53.8 \%$. There were 5 false-negatives and 1 falsepositive result, sensitivity was $80 \%$, specificity was $92.8 \%$ and accuracy was $84.5 \%$. Conclusions: Intraoral US was quite sensitive in the diagnosis of peritonsillar abscesses when performed by an experienced radiologist. Specificity was higher for transcutaneous US compared to intraoral US. However, when transcutaneous US was performed in patients with trismus, it was able to diagnose all peritonsillar abscesses, since they were large collections which are common in patients with trismus. These exams showed similar accuracy.
\end{abstract} MUSP.

${ }^{4}$ Assistant Physician - PhD - Department of Otorhinolaryngology - University Hospital - Medical School of the University of São Paulo. Head of the Otorhinolaryngology Emergency Room - HCFMUSP.

${ }^{5}$ MD. PhD, Assistant physician - Department of Radiology - Medical School of the University of São Paulo. Radiologist.

Study carried out at the Otorhinolaryngology Department and Radiology Department of the University Hospital - Medical School of the University of São Paulo. Mailing address: Dr. Bernardo Cunha Araujo Filho - Rua Elias João Tajra 1260/1500 Teresina Piauí 64049-300. E-mail: bcaf@terra.com.br Paper submitted to the ABORLPaper submitted to the ABORL-CCF SGP (Management Publications System) on January 5th, 2006 and accepted for publication on April 3rd, 2006. 


\section{INTRODUCTION}

The peritonsillar space is located between the palatine tonsil fibrous capsule (medially) and the fascia of the superior constrictor muscle (laterally), being the most common site of abscess formation in the head and neck ${ }^{1}$. It is typically more common in adolescents and young adults resulting from propagation of tonsillar infections, which lead to cellulitis or peritonsillar abscesss ${ }^{2}$. If treated incorrectly, the abscess may cause severe consequences for patients such as aspiration and pneumonia, as well as deep cervical infection with serious consequences, such as mediastinitis, sepsis and even morte ${ }^{1,3-5}$. Clinically, peritonsillar abscesses and cellulitis have a similar presentation that is almost impossible to differentiate based on the clinical history and the physical examination ${ }^{3,6,7}$. Differentiation between these two entities, which are part of the same illness, is essential for successful treatment. Peritonsillar abscesses (PAs) may be treated with needle aspiration, drainage of pus or tonsillectomy, while cellulitis (PC) is treated with antibiotics ${ }^{3,7,8}$. The differential diagnosis between cellulitis and peritonsillar abscesses is made by needle aspiration and careful aspiration of the peritonsillar space $^{8,9}$. Frequently repeated needle aspiration is needed to locate the possible abscess. This procedure is painful and risky, there is the possibility of injuring blood vessels such as the internal carotid artery, and it may be difficult in children and patients with significant trismo ${ }^{1,8,10,11}$. An abscess may not be diagnosed in some patients, which results in inadequate treatment ${ }^{7}$. Ultra-sound (US) has been used in the diagnosis of abscesses since 1950; in past 15 years it has become much more frequently used in medical conditions.

In this context there have been attempts to develop and evaluate methods to make the correct differential diagnosis between PC and PA. There are references in literature on the use of intra-oral and transcutaneous US to differentiate PC or PA, since there is no correlation between the onset of the abscess and the duration of infecção ${ }^{12}$, however these studies had a limited number of patients and inexperienced radiologists to diagnose peritonsillar space infections. A comparison between intra-oral and transcutaneous US in the differential diagnosis of PC and PA has not yet been done.

The aim of this study was to establish the accuracy, specificity and sensitivity of intra-oral and transcutaneous US in the diagnosis of cellulitis and peritonsillar abscesses.

\section{CASES AND METHODS}

In this prospective study, thirty nine patients with a clinical diagnosis of cellulitis or peritonsillar abscess were attended at the emergency unit of the Clinical Hospital of the Sao Paulo University Medical School. The research protocol was approved by the Research Ethics Committee; participants read and signed a free and informed consent form. Twenty four were women and fifteen were men aged between 7 and 44 years. Following otorhinolaryngological evaluation, all patients underwent intra-oral and transcutaneous US by a radiologist familiar with the radiological diagnosis of this entity. The radiologist did not have access to the clinical hypothesis of cellulitis or abscess raised by the otorhinolaryngologist. We used a General Electric 500 ultrasound equipment (Milwakee, USA) with a 7,5Mhz central frequency linear transducer placed on the angle of the lower jaw of the patient in orthostatism and lateral rotation of the head (see Figure 1). Intra-oral US was done with a condom-covered $6,5 \mathrm{Mhz}$ intracavity transducer covered; the patient was seated with the mouth open and xylocaine $10 \%$ spray was applied to the oropharynx for anesthesia, so that the intracavity transducer could be placed over the affected tonsil (see Figure 2).

The patients were classified, according with ultrasonographic findings, as having cellulitis or a peritonsillar abscess, and the abscess volume was measured. The diagnosis was confirmed in all patients by needle aspiration with a jelco 14 needle on three points: the superior polar region, the middle polar region and the inferior polar region. If needle aspiration was positive, an incision and drainage were undertaken. If negative, the patients were diagnosed as having cellulitis and treated with antibiotics. Intra-oral and transcutaneous US were compared with needle aspiration results (see Table 1). We calculated the sensitivity, specificity, the negative predictive value and the positive predictive value of the two tests. We also made Receiver Operator Characteristic (ROC) curves of these tests and calculated the areas under the curves (accuracy). These areas were compared to check whether a test was more accurate that the other by using the ChiSquared test.

\section{RESULTS}

Intra-oral US could not be done in 4 patients out of 39 patients due to significant trismus. Intra-oral US found abscesses in $65.7 \%$ of cases and cellulitis in $34.3 \%$ of cases. Jelco needle aspiration was positive in 21 patients and negative in 14 patients. There were 3 false positive cases and 1 false negative case. Sensitivity was $95.2 \%$ and specificity was $78.5 \%$. The positive predictive value was $87 \%$ and the negative predictive value was $91.7 \%$ (see Table 2). Transcutaneous US was done in all patients and diagnosed peritonsillar abscesses in $53.8 \%$ of cases. There were 5 false negative cases and 1 false positive case; sensitivity was $80 \%$ and specificity was $92.8 \%$ (see Table 3 ). The accuracy of transcutaneous US was $84.5 \%$ and the accuracy of intra-oral US was $86.9 \%$, with no statistically significant difference $(p=0.72)$ (Chart I). There were bilateral abscesses in 1 case. 


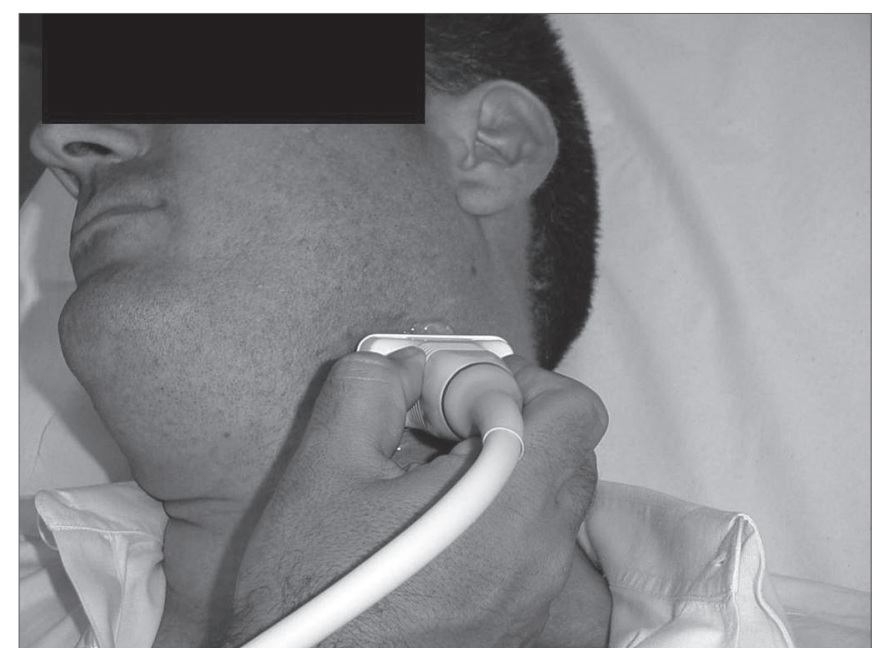

Figure 1. Transcutaneous US placed in the angle of the lower jaw with the patient in orthostatism and lateral rotation of the head.

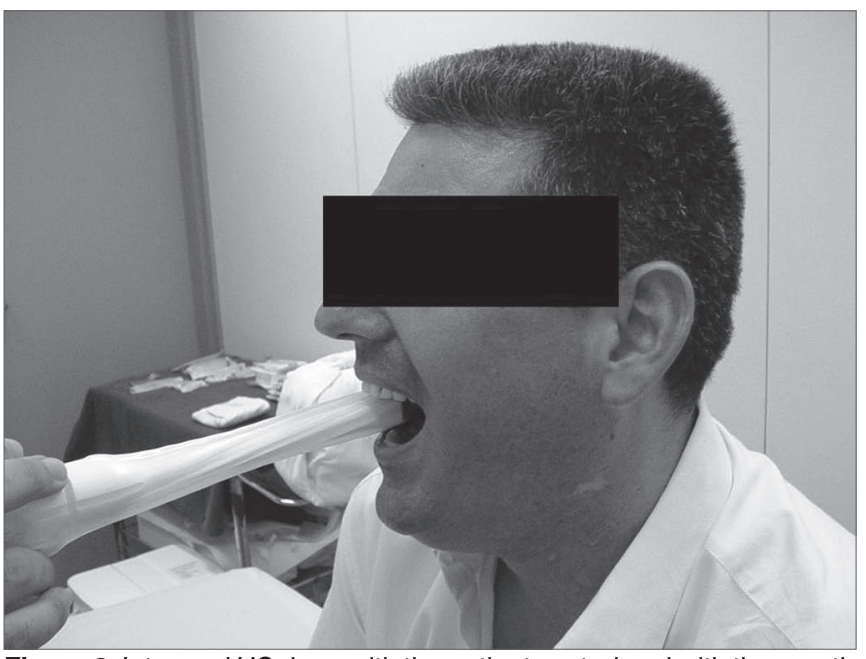

Figure 2. Intra-oral US done with the patient seated and with the mouth open, allowing contact between the intra-cavity transducer and the affected tonsil.

\section{DISCUSSION}

The origin of peritonsillar space inflammation is controversial; according to some authors, it initiates with infection of the Weber glands in the supra-tonsillar fossa1. Patients with peritonsillar abscess and cellulitis may present throat pain, fever, dysphagia, trismus, malaise and may progress unfavorably, developing aspiration pneumonia and extension of the infection into deep neck spaces, even leading to death in some cases ${ }^{1,6,7}$. Increases in the number of infections of the peritonsillar space have been observed in children due to the inadequate use of antibiotics ${ }^{11}$. Wide bore needle aspiration has been used to differentiate between PC and PA; it is a potentially dangerous, invasive and painful method ${ }^{8,10}$. Haeggstrom et $a .^{8}$ and Amhed et
Table 1. Results of abscess and cellulitis assessment in patients undergoing intra-oral US, transcutaneous US and needle aspiration.

\begin{tabular}{|c|c|c|c|}
\hline Patient & Intra-oral US & $\begin{array}{c}\text { Transcutaneous } \\
\text { US }\end{array}$ & $\begin{array}{c}\text { Needle } \\
\text { aspiration }\end{array}$ \\
\hline 1 & Abscess & Abscess & Abscess \\
\hline 2 & Abscess & Abscess & Abscess \\
\hline 3 & Not done (trismus) & Abscess & Abscess \\
\hline 4 & Cellulitis & Cellulitis & Cellulitis \\
\hline 5 & Abscess & Abscess & Cellulitis \\
\hline 6 & Abscess & Abscess & Abscess \\
\hline 7 & Abscess & Abscess & Abscess \\
\hline 8 & Cellulitis & Cellulitis & Cellulitis \\
\hline 9 & Cellulitis & Cellulitis & Cellulitis \\
\hline 10 & Abscess & Cellulitis & Cellulitis \\
\hline 11 & Cellulitis & Cellulitis & Cellulitis \\
\hline 12 & Abscess & Abscess & Abscess \\
\hline 13 & Abscess & Abscess & Abscess \\
\hline 14 & Abscess & Abscess & Abscess \\
\hline 15 & Abscess & Cellulitis & Abscess \\
\hline 16 & Abscess & Abscess & Abscess \\
\hline 17 & Not done (trismus) & Abscess & Abscess \\
\hline 18 & Cellulitis & Cellulitis & Cellulitis \\
\hline 19 & Cellulitis & Cellulitis & Cellulitis \\
\hline 20 & Abscess & Cellulitis & Abscess \\
\hline 21 & Not done (trismus) & Abscess & Abscess \\
\hline 22 & Not done (trismus) & Abscess & Abscess \\
\hline 23 & Cellulitis & Cellulitis & Cellulitis \\
\hline 24 & Cellulitis & Cellulitis & Abscess \\
\hline 25 & Abscess & Abscess & Abscess \\
\hline 26 & Abscess & Abscess & Abscess \\
\hline 27 & Abscess & Abscess & Abscess \\
\hline 28 & Abscess & Cellulitis & Abscess \\
\hline 29 & Abscess & Abscess & Abscess \\
\hline 30 & Abscess & Abscess & Abscess \\
\hline 31 & Cellulitis & Cellulitis & Cellulitis \\
\hline 32 & Cellulitis & Cellulitis & Cellulitis \\
\hline 33 & Cellulitis & Cellulitis & Cellulitis \\
\hline 34 & Abscess & Cellulitis & Cellulitis \\
\hline 35 & Abscess & Abscess & Abscess \\
\hline 36 & Abscess & Cellulitis & Abscess \\
\hline 37 & Abscess & Abscess & Abscess \\
\hline 38 & Cellulitis & Cellulitis & Cellulitis \\
\hline 39 & Abscess & Abscess & Abscess \\
\hline
\end{tabular}


Table 2. Intra-oral US

\begin{tabular}{lcc}
\hline & Positive Negative & Total \\
\hline Abscess & $20(87 \%) 01(8,3 \%)$ & $21(60 \%)$ \\
Cellulitis & $03(13 \%) 11(91,7 \%)$ & $14(40 \%)$ \\
Total & $23(100 \%) 12(100 \%)$ & $35(100 \%)$ \\
\hline
\end{tabular}

Table 3. Transcutaneous US. Aspiration with jelco

\begin{tabular}{lcc}
\hline & Positive Negative & Total \\
\hline Abscess & $20(95,2 \%) 05(27,7 \%)$ & $25(64,1 \%)$ \\
Cellulitis & $01(4,8 \%) 13(72,3 \%)$ & $14(35,9 \%)$ \\
Total & $21(100 \%) 18(100 \%)$ & $39(100 \%)$ \\
\hline
\end{tabular}

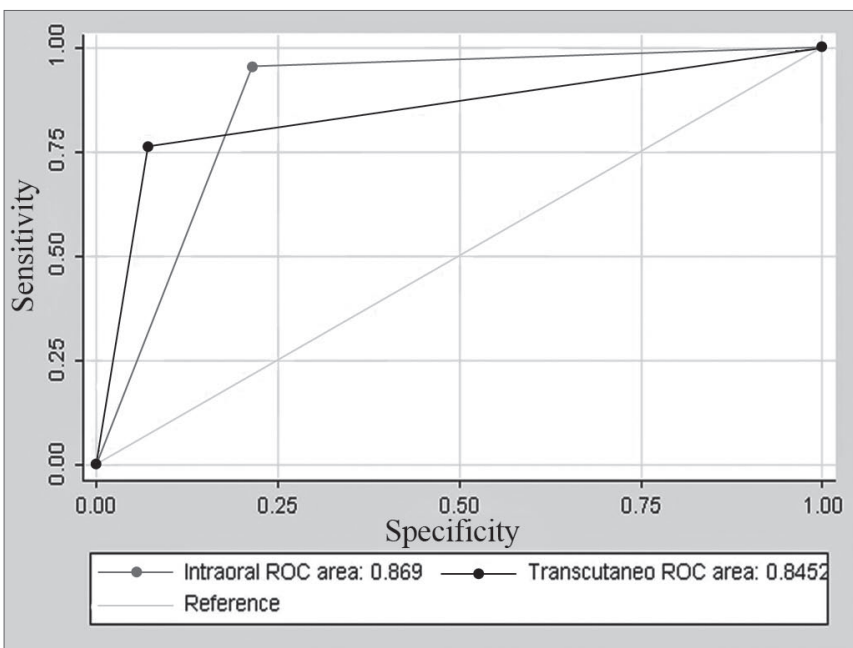

Chart 1. ROC (Receiver Operator Characteristic) curve for Intra-oral and Transcutaneous US .

al. ${ }^{9}$ have suggested that needle aspiration is an accurate procedure for this diagnosis, in contrast to Scott et al. ${ }^{7}$, that reached $89 \%$ sensitivity compared with computerized tomography. Computerized tomography is effective to diagnose deep neck affections, however, it is expensive, subject to complications due to use of contrast and difficult to access in emergency units ${ }^{1,3}$. We believe that systematic punctures of the superior, middle and inferior polar region of the tonsillar fossa may reduce false negatives; furthermore, in a clinical setting, this method has been effective to diagnose infection in the peritonsillar space. Miziara et $\mathrm{al}^{3}{ }^{3}$, in attempts to reduce the risks of unnecessary needle aspiration in patients, have evaluated the use of intra-oral US to diagnose abscesses and cellulitis, with a $92.3 \%$ sensitivity and a $62.5 \%$ specificity. Haeggstrom et $\mathrm{al}^{8}{ }^{8}$ reported an $85 \%$ success rate in the diagnosis of abscesses with intra-oral US. In our study, intra-oral US sensitivity was $95.2 \%$ and specificity was $78.5 \%$, superior to the abovementioned studies. A larger sample size and an experienced radiologist were determining factors for the success of intra-oral US in differentiating between cellulitis and abscesses. Analyzing the 3 false positive cases and 1 false negative with intra-oral US, these occurred with small collections of liquid $(<1 \mathrm{ml})$, similar to Ahmed ${ }^{9}$ e Strong ` $s^{6}$ findings. Intense edema and inflammation may also lead to false positive results. Intra-oral US could not be done in 4 patients that had significant trismus. The sensitivity of transcutaneous US (80\%) was lower compared to intraoral US (92.5\%) in the same patients, however it was more specific (92.8\%) compared to intra-oral US (90\%). Ahmed et al. ${ }^{9}$, in a study of 27 patients, reported a $90 \%$ sensitivity for transcutaneous US, better than our results, however inferior to the rate obtained with intra-oral US. Transcutaneous US had 5 false negative results, limiting its use to the diagnosis of peritonsillar abscesses. Small needle-aspirated collections of liquid $(<2.5 \mathrm{ml})$ is also related to an increase in false negatives during transcutaneous US. We recommend that results of this exam be evaluated with care, as there was a tendency to underestimate the volume of collections of liquid. When intra-oral US could not be used due to trismus, transcutaneous US diagnosed abscesses in 100\% of cases. The aspirated volume was increased in these patients ( $>4 \mathrm{ml}$ ), which is related to the more exuberant clinical picture and which was responsible for the increased sensitivity of transcutaneous US in these patients. In literature unilateral abscesses are observed in 93\% of cases $^{2,3}$, similar to our percentage. Transcutaneous US was important in cases where intra-oral US was impossible. In our study transcutaneous US and intra-oral US together improved the diagnosis of PA and PC compared to Miziara et al. $` s^{3}$ and Haeggstrom et al. $` s^{8}$ rates. US also allowed the otorhinolaryngologist to guide needle puncture, avoiding multiple blind punctures in any one patient. This has also been credited to US by Sakagushi et al. ${ }^{1}$, Patel et al. ${ }^{4}$ and Blaivas et al..$^{5}$ Although transcutaneous US was specific, some cases were not detected. When dealing with patients that have potential risks of serious complications, high false negative rates are unacceptable. A sensitive low false negative rate examination is needed for the diagnosis of peritonsillar space conditions. Transcutaneous US, a more specific exam, could be in cases where intra-oral US was impossible; used simultaneously, they would not increase diagnostic accuracy in peritonsillar infections.

\section{CONCLUSION}

Intra-oral US was more sensitive but less specific than transcutaneous US in the diagnosis of peritonsillar abscesses, when undertaken by an experienced radiologist. It is an efficient and accurate method to differentiate the diagnosis of cellulitis and peritonsillar abscesses. Only transcutaneous US was possible in patients with trismus, being sufficiently sensitive in larger collections of liquid, usually seen in these patients. These examinations had statistically similar accuracy. 


\section{REFERENCES}

1. Sakagushi M, Sato S, Asawa S. Computed tomographic findings in peritonsillar abscess and cellulitis. The Journal of Laryngology and Otology. 1995;109:449-51.

2. Ophir D, Poria Y, Marshak G. Peritonsillar abscess. A prospective evaluation of outpatient management by needle aspiration. Arch Otolaryngology Head and Neck Surgery 1988;114:661-3.

3. Miziara ID, Koishi HU, Zonato AI, Miniti A, De Menezes MR. The use of ultrasound evaluation in the diagnosis of peritonsillar abscess. Rev Laryngol Otol Rhinol 2001;122:201-3.

4. Patel KS, Ahmad S, Michel M. The role of computed tomography in the management of peritonsillar abscess. Otolaryngol Head and Neck Surgery 1992;107:727-32.

5. Lyon M, Glisson P, Blaivas M. Bilateral Peritonsillar Abscess Diagnosed on the Basis of Intra-oral Sonography. Journal Ultrasound of Medicine 2003;22(9):993-6.

6. Strong EB, Woodward PJ, Johnson LP. Intra-oral Ultrasound Evaluation of Peritonsillar Abscess. Laryngoscope 1995;105:779-82.
7. Scott PMJ, Loftus WK, Ahuja A, Van Hasselt CA. Diagnosis of Peritonsillar Infections: A Prospective Study of Ultrasound Computerized Tomography and Clinical Diagnosis. The Journal of Laryngology and Otology 1999;113:229-32.

8. Haeggstrom A, Gustaffson O, Engquist S. Intra-oral ultra-sonography in the diagnosis of peritonsillar abscess. Otolaryngol Head and Neck Surgery 1993;108:243-47.

9. Ahmed K, Shah K, Jones AS, Smethurst A. The Role of Ultrasound in the Management of Peritonsillar Abscess. The Journal of Laryngology and Otology 1994;108:610-2.

10. Kornblut AD. Peritonsillar cellulitis and abscess: current considerations in diagnosis and treatment. American Journal of Emergency Medicine 1987; 5:258-60.

11. Garcia Callejo FJ, Velert Vila MM. Our Experience in the Management of Peritonsillar Phlegmon and Abscess in Children. An Esp Pediatr 1999;51(3):251-6.

12. Kew J, Ahuja A, Loftus WK, Scott PM, Metreweli C. Peritonsillar Appearance on Intra-oral Ultrasonography. Clin Radiol 1998;53 (2):1436. 